\title{
Les éditeurs de littérature française aux États-Unis et en Amérique latine durant la Deuxieme Guerre mondiale
}

Jacques Michon ${ }^{\dagger}$

L'Occupation de la France par l'armée allemande, de juin I940 à août 1944, a provoqué l'émigration de nombreux intellectuels et l'implantation de maisons d'édition françaises en dehors de l'hexagone. Plusieurs entreprises de publications ont alors été créées en Suisse, en Afrique du Nord, au Moyen-Orient, en Amérique du Nord et du Sud. Nous voulons présenter ici les principaux éditeurs nordet sud-américains qui sont apparus dans ce contexte historique. Ces nouvelles maisons avaient surtout pour objectif d'assurer la diffusion de la pensée française dans le monde. Elles rééditaient les classiques de la littérature et réimprimaient les titres en demande. De plus, les écrivains exilés à New York, à Montréal, à Mexico, à Rio de Janeiro et à Buenos Aires trouvaient là un débouché pour leurs ouvrages.

Nous aborderons ici dans ses grandes lignes les caractéristiques de la production littéraire de quelques-unes de ces entreprises. Si elles entretenaient entre elles des rapports étroits de collaboration par le biais de la co-édition et d'ententes de distribution, plusieurs se faisaient une vive concurrence. Les gouvernements en guerre et les organismes de propagande anti-fasciste, comme l'Office of War Information, jouèrent aussi un rôle dans le développement et le soutien de leurs activités. ${ }^{\text {I }} \mathrm{Si}$ la fin de la guerre provoqua la disparition de la plupart d'entre elles, ces maisons nées en I 940 laissèrent

† Jacques Michon est professeur au Département de lettres et communications de 1'Université de Sherbrooke où, depuis 1982, avec quatre autres collègues, il dirige un groupe de recherche sur l'édition littéraire au Québec. Il a fait paraître récemment deux ouvrages en collaborataion édités aux Presses de l'Université Laval: L'édition littéraire en quête d'autonomie, Albert Lévesque et son temps (I994) et Édition et pouvoirs (I995). Ce texte constitue la version française d'une communication intitulée "Canadian, American, and Latin American Publishers of French Works during World War II", présentée au dernier congrès de SHARP (Society for the History of Authorship, Reading and Publishing) à Washington, I4-I 6 juillet I994. 
derrière elles un héritage intellectuel et social dont on n'a pas fini de mesurer la valeur et la portée.

Nous n'avons pas le temps d'aborder ici toute la production de l'époque qui comprend plusieurs milliers de titres ni de présenter toutes les entreprises qui ont participé de près ou de loin à ce mouvement éditorial. Nous mettrons d'abord l'accent sur les maisons les plus en vue qui indiquent les tendances et une évolution caractéristiques de l'ensemble.

Cette étude s'inscrit dans le cadre d'un projet de recherche sur l'histoire de l'édition francophone au $\mathrm{xx}^{\mathrm{e}}$ siècle menée par l'équipe des chercheurs de 1'Université de Sherbrooke. ${ }^{2}$ C'est l'étude de l'édition durant la Deuxième Guerre mondiale qui nous a mis sur la piste de ces éditeurs francophones de l'Amérique du Nord et du Sud. Les documents imprimés et les archives des auteurs et éditeurs québécois consultés et colligés au cours de cette recherche nous ont amené à mesurer l'ampleur et l'importance du phénomène qui couvre une décennie, r940-49. Cette documentation nous a permis d'identifier plusieurs maisons d'édition, d'établir le catalogue de leurs publications et de dégager leurs réseaux de relations sur le plan international. Une étude des archives des maisons situées aux États-Unis et en Amérique latine dépasse actuellement nos moyens $\mathrm{d}$ 'investigation. Une telle enquête fournirait sans doute des informations intéressantes sur les propriétaires des entreprises et sur leur mode de gestion et de distribution. Le présent travail s'appuie sur des catalogues reconstitués à partir de différents documents imprimés, prière d'insérer et publicité, et de quelques pièces d'archives disponibles dans les fonds de Bernard Valiquette à la Bibliothèque nationale du Québec. Les exemplaires retracés dans les bibliothèques et chez certains libraires d'occasion nous ont permis de compléter ces informations. Avant d'aborder directement les maisons d'édition de New York et d'Amérique latine, il convient de dire quelques mots sur l'édition à Montréal durant cette période.

En 1940, Montréal constitue le centre de production du livre francophone le plus important dans les trois Amériques. Une population de quatre millions d'habitants de langue maternelle française assure à cette industrie un marché stable et important sans équivalent sur le continent. Si l'édition littéraire de langue française est déjà bien développée avant I930, la guerre vient stimuler la production du fait de l'interruption des relations commerciales avec la France après l'Occupation. Plusieurs nouvelles maisons sont créées entre I940 et I 945 pour répondre aux besoins en livres 
français dans le monde libre. La production connaît alors un essor spectaculaire $(+244 \%){ }^{3}$

Je ne m'attarderai pas sur ce phénomène qui a déjà fait l'objet de plusieurs publications. ${ }^{4}$ Je me contenterai de signaler ici quelques chiffres. Sur environ 4000 titres publiés de 1940 à 1949 , près du tiers $(27 \%)$ de la production est constitué, de 1943 à I 947 , de rééditions et de réimpressions de titres français épuisés ou qui ne sont plus disponibles en Amérique du Nord. ${ }^{5}$ En 1944, on compte plus de vingt-cinq maisons d'édition littéraire, huit d'entre elles produisant $82 \%$ de tous les ouvrages; certaines maisons dans les années les plus fastes peuvent faire paraître jusqu'à une centaine de titres par année. En I945, le nombre d'exemplaires produits depuis le début de la guerre est estimé à cinq millions. ${ }^{6}$

Bien que moins importantes que celles de Montréal en terme de production, les maisons de New York et d'Amérique latine ont aussi joué un rôle dans la diffusion et la promotion du livre français. Leur situation géographique respective, l'existence de communautés françaises et francophiles importantes et la dispersion des auteurs sur les deux continents favoriseront la création de nouvelles maisons. Nous allons nous attarder ici aux principaux éditeurs de ces deux espaces américains et latino-américains qui sont, en ce qui nous concerne, les Éditions de la Maison française, les Éditions françaises de Brentano's, Ediciones Quetzal, Americ-Edit et Atlantica Editora.

On sait que, pendant cet essor éditorial, en France même, l'édition, bien que surveillée par l'occupant et souvent censurée, était encore bien vivante. ${ }^{7}$ Ce sont les difficultés d'exportation du livre, surtout liées au contingentement du papier et aux problèmes de communications transatlantiques, qui rendaient nécessaires la création de nouvelles maisons à l'étranger et en particulier en Amérique du Nord où la demande en livres français était importante.

\section{Les maisons de New York: EMF et Brentano's}

À New York, plusieurs maisons firent de l'édition en français durant les années 40: Brentano's, les Éditions de la Maison française, Didier et Pantheon Books. ${ }^{8}$ Elles étaient dirigées par des intellectuels français exilés aux États-Unis. Les deux plus importantes, Brentano's et les Éditions de la Maison française (EMF), publièrent ensemble près de 300 ouvrages (voir Tableau I). Elles faisaient paraître d'abord des nouveautés et n'accordaient que peu de place 
aux réimpressions, laissant cela aux éditeurs canadiens qui avaient des ressources et un cadre législatif qui le permettait. ${ }^{9}$ Elles publiaient d'abord des ouvrages d'écrivains établis temporairement en Amérique du Nord. ${ }^{\text {ro }}$

Les Éditions de la Maison française ont été fondées à l'automne de I940. ${ }^{\text {II }}$ La note liminaire du premier titre paru, Tragédie en France d'André Maurois, situait bien les intentions de la direction qui voulait exprimer le point de vue français sur les événements que venait de connaître la France. ${ }^{\mathrm{I2}}$ Il s'agissait de rectifier les erreurs et les fausses idées véhiculées par la presse. Dans ses mémoires, André Maurois exprime les sentiments qui l'animent à cette époque:

\begin{abstract}
Avec tristesse et surprise, nous constations le développement d'une campagne, d'origine fort suspecte, écrit-il, qui accusait nos soldats de ne s'être pas défendus. Or j'avais vu nos armées, nos chefs; je connaissais les fautes, mais aussi les vertus; je savais que le mal avait été, non dans le manque de courage, mais dans le manque de préparation et que, si l'on cherchait les responsables, on les trouverait en Angleterre et en Amérique autant qu'en France. D'où l'idée que je devais dire, aussi exactement que j'en serais capable, ce que $j^{\prime}$ avais vu de cette guerre et exposer ce qu'avaient été, à mon avis, les causes du désastre. J'écrivis en hâte quelques articles, qui furent ensuite réunis en volume sous le titre de Tragédie en France. ${ }^{\mathrm{I3}}$
\end{abstract}

Une grande partie de la production des EMF a pour but de faire connaître aux Nord-Américains et aux Français de la diaspora le point de vue des écrivains et des intellectuels, témoins en première ligne de la débâcle en Europe de l'Ouest. La maison fait paraître plus de I 53 titres de I940 à I 947 et dans plusieurs collections consacrées à l'actualité européenne. Les auteurs tentent d'expliquer aux étrangers la situation sur le terrain, de documenter les travaux des professionnels de l'information et de procurer aux dirigeants des arguments susceptibles de déboucher sur des solutions politiques. De l'écrit de combat à l'étude savante, en passant par les récits vécus, ces ouvrages étaient destinés au grand public et aux faiseurs d'opinion (journalistes, intellectuels, professeurs et hommes politiques).

Trois collections permettaient à l'éditeur de cibler ces différents groupes de lecteurs. ${ }^{\mathrm{I}}$ "Voix de France" publiait des essais et des romans; la collection "Civilisation", dirigée par Jacques Maritain, faisait place aux écrits politiques des professeurs de l'École des 
hautes études de New York; ${ }^{15}$ la collection “De Cap et d'Épée» était consacrée aux romans d'espionnage de Robert Goffin racontant les aventures sensationnelles du détective Merchandon aux prises avec les agents de la Gestapo. ${ }^{16}$ Ici la fiction rejoint la littérature militante. Parus en I94I et 1942, ces romans tentent de fournir aux lecteurs franco-américains, par l'argumentation et le pathos, de bonnes raisons d'encourager leur pays à s'engager dans la guerre contre 1'Allemagne.

À côté de ces publications et des écrits de circonstance destinés à convaincre les lecteurs du bien fondé d'une intervention des alliés en Europe, il faut mentionner aussi la publication d'oeuvres moins directement reliées aux événements et signées par des auteurs domiciliés aux États-Unis. Les EMF font paraître la suite des Hommes de bonne volonté (vol. I 9 à 22) de Jules Romains, Varouna (I94I) de Julien Green, Pilote de guerre (I942) de Saint Exupéry, ${ }^{17}$ La grande clarté du Moyen-Age (1943) de Gustave Cohen, La farce est jouée, vingt-cinq ans de théâtre en France (1942) de Maurice Coindreau, Le Classicisme français (1 942) de Henri Peyre, L'histoire merveilleuse de la Louisiane française (I94I) de Régine HubertRobert, La pensée de Saint-Paul (I94I) de Jacques Maritain, et Les Grandes amitiés (2 vol.: I94I, I944) de Raïssa Maritain.

Les Éditions françaises de Brentano's ont commencé leurs activités en I94I. ${ }^{18}$ Propriété de la librairie Brentano's de New York, reconnue, entre autres, pour sa section de livres étrangers, en I945, la maison poursuit les objectifs suivants:

Le premier: ne rien négliger de ce qui pourrait être utile pour entretenir $\mathrm{l}^{\prime}$ attachement à la culture française $[\ldots]$.

Le second: à l'heure où en France la liberté de penser était étouffée, permettre à toutes les opinions de s'exprimer $[\ldots]$.

Le troisième: permettre la diffusion des livres de tous les genres. [...]

Le quatrième: donner aux livres une forme plaisante et attrayante, à la fois par le choix des couvertures et par la typographie. ${ }^{\text {I9 }}$

La maison reste fidèle à ces principes en publiant, jusqu'en I947, plus de I 36 titres de 90 auteurs différents et dans tous les genres. Comme les EMF, Brentano's publie des ouvrages de littérature générale. Elle fait paraître aussi bien des romans populaires de Maurice Dekobra et de Michel Georges-Michel, des livres pour enfants (les récits de la Comtesse de Ségur), des écrits divers sur l'actualité que des essais philosophiques et des études d'économie 
politique. En 1943, "La Bibliothèque Brentano's”, une collection placée sous le patronage de professeurs d'université, est lancée pour promouvoir les études historiques, économiques et sociales consacrées aux problèmes de l'après-guerre. ${ }^{20}$ L'éditeur entretient aussi des rapports avec l'avant-garde littéraire. André Breton, le chef de file du surréalisme, y fait paraître la première édition commerciale d'Arcane 17 qu'il avait rédigé au Québec (à Percé et Sainte-Agathe du 20 août au 20 octobre 1944$).{ }^{21}$ Breton participe également avec Marcel Duchamp en I 943 à la décoration d'une vitrine de la grande librairie de la Cinquième Avenue consacrée à la parution de La part du diable de Denis de Rougemont. ${ }^{22}$

Des ouvrages publiés à Montréal par les Éditions Variétés et Bernard Valiquette figurent également au catalogue de Brentano's à hauteur de $23 \%$ (3I/I 36 titres). ${ }^{23}$ En contrepartie, les éditeurs montréalais distribuent les livres de Brentano's au Canada. On peut voir un exemple de cette collaboration dans les deux tirages des Lauriers sont coupés . . . de Margaret Hughes (voir l'illustration). ${ }^{24}$ Le marché québécois constituait un débouché important pour les titres français des éditeurs comme nous le verrons plus loin.

$\mathrm{Si}$ on tente de tracer les grandes lignes qui se dessinent chez ces deux éditeurs, on constate que la littérature et l'histoire constituent les catégories les plus importantes avec une légère prédominance du roman aux EMF et de l'essai d'actualité chez Brentano's (Tableau I). La littérature pour la jeunesse chez Brentano's et la philosophie aux EMF constituent des spécialités propres à chacune de ces maisons. La part des nouveautés est dans l'ensemble importante, $96 \%$ aux EMF et $77 \%$ chez Brentano's.

Sur le plan idéologique, Brentano's apparaît comme un éditeur plus libéral que son concurrent. Il apporte son appui au surréalisme; publie des études savantes, peu rentables, destinées à un public restreint; et son intérêt pour la littérature canadienne, fruit de ses rapports avec les éditeurs de Montréal, témoigne d'une ouverture et d'un intérêt pour une littérature en émergence.

Au contraire, les EMF, marqués par la figure de Jacques Maritain, philosophe catholique et militant gaulliste, offrent une image plus traditionnelle avec des auteurs comme Jules Romains (I 5 livres), André Maurois (II), Robert Goffin (8), Michel Georges-Michel (5) et Emil Ludwig (5). Jacques Maritain est sans doute l'auteur le plus important de la maison dans la mesure où il signe onze livres, écrit plusieurs préfaces et dirige la collection "Civilisation". C'est lui également qui rédige la présentation du catalogue général des EMF en $1944 . .^{25}$ 
Quelques auteurs seulement (une dizaine) se retrouvent simultanément chez les deux éditeurs. ${ }^{26}$ Dans l'ensemble, chaque éditeur a ses vedettes attitrées. ${ }^{27} \mathrm{Au}$ total, on dénombre chez les deux éditeurs réunis, une population de 157 auteurs, poètes, romanciers, journalistes, professeurs, diplomates, hommes politiques qui, pour la plupart, retourneront dans leur pays d'origine après la Libération. ${ }^{28}$

Comme ces ouvrages s'adressent d'abord au marché francophone, les livres sont tous brochés à la française, ce qui les distinguent de la production courante américaine reliée. Certaines couvertures des EMF rappellent volontairement celles des éditions Gallimard, allant jusqu'à imiter dans le design de l'acronyme emf celui de la célèbre nrf (Nouvelle revue française). Si les lecteurs visés sont, à l'origine, les Français de la diaspora, les professeurs et les étudiants des collèges et des universités américaines, les lecteurs francophones du Québec se révéleront à terme plus nombreux, comme le constate John Tebbel qui aborde le cas de Didier dans son histoire de l'édition aux États-Unis:

There was an active demand in North America for French titles, but most of the market was in French Canada, and it was there that Didier concentrated his advertising, with the result that 85 percent of his sales were made in the Canadian market. ${ }^{29}$

Avec la libération progressive des colonies françaises de l'Afrique, les livres français produits à Montréal et à New York traversent $1^{\prime}$ Atlantique en plus grand nombre. ${ }^{3 \circ}$ Les achats se font avec l'aide financière de l'Office of War Information qui émet les permis nécessaires pour le transport des livres et le transfert des paiements en dollars américains. ${ }^{3 \mathrm{I}}$ Les commandes passent par New York et les Messageries Hachette, représentées par les propriétaires des EMF, la French \& European Publications Inc. ${ }^{32}$

\section{Les éditeurs français d'Amérique latine}

L'Amérique latine prend aussi part à cette activité de production et de diffusion du livre français. Plusieurs maisons d'édition, à la même époque, font leur apparition à Mexico, à Rio de Janeiro et à Buenos Aires (voir Tableau III). Créées par des intellectuels français réfugiés dans ces régions, elles s'adressent sensiblement aux mêmes lecteurs. Sur ces éditeurs nos renseignements sont beaucoup plus 
fragmentaires. Nous avons pu identifier six entreprises, une à Mexico, deux à Rio et trois à Buenos Aires.

La maison de Mexico, Ediciones Quetzal, 33 créée par Michel Berveiller en I94I, publie des auteurs classiques (Molière, Diderot, Voltaire, Musset, Baudelaire), des traductions espagnoles d'ouvrages français et fonde la collection "Renaissance», dirigée par René Etiemble, alors professeur à l'Université de Chicago, ${ }^{34}$ où paraissent successivement Le Petit Bois et autres contes (1942) de Jules Supervielle et $L$ a communion des forts (1943) de Roger Caillois, ${ }^{35}$ auteurs établis respectivement à Montévideo et à Buenos Aires. La maison change de propriétaire en 1942 lorsque Berveiller quitte la direction pour joindre les forces de De Gaulle à Londres. Etiemble a de nombreux projets. Il veut faire paraître des livres de Henri Focillon, de Denis de Rougement, de Georges Bernanos et de Maurice Coindreau. Mais ces projets sont abandonnés lorsqu'il quitte l'Amérique pour 1'Afrique du Nord en janvier $1944 \cdot{ }^{36}$ Nous perdons la trace de l'éditeur après I945. Nous savons cependant que ses livres étaient distribués au Canada par les Éditions Bernard Valiquette de Montréal. ${ }^{37}$

Le Brésil est aussi une terre d'accueil pour les écrivains européens parmi lesquels on retiendra, entre autres, les noms de Georges Bernanos et de Stefan Zweig. A Rio, deux maisons publient des livres en français, Americ-Edit et Atlantica Editora. La première semble la plus importante. Dirigée par un écrivain français en exil, Max Fischer (1880-1957), la Société Americ-Edit ${ }^{38}$ fait surtout de la réédition d'ouvrages français contemporains, épuisés ou non disponibles en Amérique. Nous avons dénombré une centaine de titres au catalogue parmi lesquels figurent, entre autres, les oeuvres de Paul Claudel, de Colette, d'André Gide et de François Mauriac, rééditées avec l'autorisation de Bernard Grasset et des éditions Flammarion. Ces ouvrages sont distribués à Montréal par les Éditions Variétés, maison réputée pour ses réimpressions d'auteurs français contemporains. ${ }^{39}$

Atlantica Editora, ${ }^{4 \circ}$ maison dirigée par Charles Ofaire, un éditeur suisse installé à Rio, fut, semble-t-il, une entreprise moins commerciale et plus engagée dans le combat pour la France Libre. Elle restera connue comme la maison d'édition de Georges Bernanos en Amérique latine. Sur la vingtaine de titres que nous avons relevés, publiés de $\mathrm{I} 942$ à I 945 , huit sont signés par Bernanos. L'auteur avait élu domicile au Brésil en r 938 et fut l'un des grands inspirateurs de la Résistance. En 1942, sa Lettre aux Anglais, co-éditée à Rio par Atlantica et à Montréal par les Éditions de l'Arbre, fit le tour du 
monde. ${ }^{4 \mathrm{I}}$ En 1940, mettant en veilleuse son activité de romancier, Bernanos avait commencé une longue campagne d'articles de guerre dans les journaux et les revues du Brésil, du Canada (dans La Nouvelle Relève aux Éditions de l'Arbre) et d'Angleterre. Ces articles, réunis dans une série de quatre volumes intitulés Chemin de la Croix-des-Ames, (1943-45), seront tous publiés chez cet éditeur. ${ }^{42}$

Buenos Aires est un autre foyer important de culture française en Amérique du Sud grâce, entre autres, à Victoria Ocampo et à sa revue Sur (193I-1979) qui édite Les Lettres françaises de Roger Caillois de I94I à I944. Une dizaine de livres signés par André Breton, St-John Perse, Jules Supervielle et Paul Valéry paraissent à cette enseigne durant la guerre. Une autre petite maison orientée vers une culture d'élite, les Éditions Viau, créées par un éditeur canadien, un certain Monsieur Viau sur lequel nous n'avons aucun renseignement, publie dans les années 44-45 des éditions de luxe des Chants de Maldoror de Lautréamont et les Poèmes d'Edgar Poe dans la traduction de Stéphane Mallarmé. 43

La plupart de ces maisons d'édition qui ont servi la culture française de 1940 à 1945 disparaissent au lendemain de la guerre avec le retour en France de ses auteurs ou de ses directeurs littéraires. Elles ferment leur porte, ou font faillite, au fur et à mesure de la reprise économique européenne. Cependant, durant un certain nombre d'années, de juin 1944 à l'été 1947, les éditeurs francoaméricains continuent à jouer un rôle de suppléance en attendant le rétablissement complet des relations commerciales entre l'Europe et l'Amérique. En 1945, les difficultés économiques de l'après-guerre rendent encore nécessaires les réimpressions nordaméricaines de nouveautés parisiennes. C'est ainsi que le premier Prix Goncourt de l'après-guerre, édité chez Flammarion, Mon village à l'heure allemande de Jean-Louis Bory, est réédité à New York aux EMF. ${ }^{44}$ En I947, 1'Almanach des lettres fait le constat suivant:

Après la Libération, l'impression de livres français à l'étranger a permis de décharger nos éditeurs d'une partie de leur lourd fardeau: continuation de leur activité, retard à rattraper, et surtout reconstitution des fonds. Des accords directs ont été passés et continuent de l'être. [ . . ] ll y a, assurément, à la continuation de cette politique, de graves inconvénients. Mais, pour l'instant, il ne faut pas songer tout imprimer chez nous. Notre faiblesse financière nous interdit les achats massifs de papier qui pourraient remettre à flot l'édition française; la même raison amène les producteurs de papier à freiner leur production. [ . . . ] La situation du papier — qui s'était 
sérieusement améliorée voici quelques mois - est redevenue si mauvaise, qu'il nous faudra longtemps encore profiter des facilités que nous offrent les imprimeurs étrangers. (p. I 54)

Un auteur de la nouvelle génération comme Jean-Paul Sartre qui vient à Montréal et à New York en I946, explore les possibilités de se faire éditer par les éditeurs nord-américains. ${ }^{45} \mathrm{D}$ 'autres, comme Georges Duhamel ou Léon Blum, prolongent les ententes déjà établies avec des éditeurs montréalais durant la guerre et signent des contrats pour de nouveaux titres. Dans un temps où l'approvisionnement en papier est encore difficile, il est plus avantageux pour les éditeurs français de signer des ententes de co-édition pour une diffusion sur le continent américain. ${ }^{46}$ Les éditeurs encore en place en 1945-46 signent des ententes avec les maisons parisiennes et tentent parfois de vendre une partie de leur production en France. ${ }^{47}$ Le Graphique I, qui établit les courbes de publication des EMF et de Brentano's d'après un échantillon de I 48 titres $(5 \mathrm{I} \%)$, dont nous avons pu établir la date, sur les 289 répertoriés (voir Tableau II), vient confirmer en partie l'intensification de la production des éditeurs en $1945 .{ }^{48}$ Lorsque la situation européenne se redressera, après l'application du Plan Marshall en I948, les éditeurs parisiens reprendront rapidement leur pleine activité de production et reviendront en force sur les marchés nord- et sudaméricains mettant ainsi définitivement un terme aux entreprises de guerre.

Toutes les maisons d'édition dont nous avons parlé disparaissent à la fin des années 40 . Seuls les éditeurs de Montréal qui étaient déjà en affaire avant $\mathrm{I} 939$ continuent à publier des livres français. Quatre maisons seulement (Beauchemin, Fides, Granger Frères, Éditions du Lévrier) sur les 27 qui étaient en activité au milieu des années 40 restent en affaire. Cette situation provoque au Québec une crise de l'édition qui durera plus de dix ans et qui ne sera résolue qu'en 1960 avec la Révolution tranquille et la mise en place de programmes d'aide à l'industrie du livre par les gouvernements de Québec et d'Ottawa.

Nous commençons à peine à mesurer les retombées intellectuelles de cette époque qui ont été importantes des deux côtés de l'Atlantique. Au Québec, entre autres, le contact avec un grand nombre d'intellectuels français a favorisé des échanges fructueux avec les milieux littéraires et artistiques. Les relations entre le Nord et le Sud des Amériques ont été l'occasion de découvertes culturelles réciproques: publications d'auteurs canadiens à New York 
et traduction d'auteurs nord- et sud-américains à Montréal.49 Plusieurs des oeuvres écrites ou publiées en première édition en Amérique ont été rééditées à Paris après la guerre. Il faut signaler également, de l'autre côté de l'Atlantique, la découverte des littératures américaine et sud-américaine par le public français, grâce en particulier à Maurice Coindreau et à Roger Caillois, deux exilés rapatriés en 1945. Ainsi l'édition française en Amérique n'a pas seulement été la transplantation géographique d'activités éditoriales européennes, elle a aussi été l'occasion de découvertes réciproques, d'échanges et de transactions qui ont sensiblement modifiés les relations et les perceptions des milieux littéraires de l'après-guerre des deux côtés de l'Atlantique.

\section{SUMMARY}

The German occupation of France from June 1940 until August I944 caused an emigration of a large number of French intellectuals. As a result of the occupation many publishing ventures were established in various parts of the world, especially in the United States and in South America. This paper discusses what appears to be the most important publishers during this period of exile, whose common purpose was to express the point of view of a liberated France. An attempt is made to examine the underlying characteristics of the literary production of these publishers who, in spite of differences in terms of publishing programme, travelled similar paths. The end of the war brought about the disappearance of most of these expatriate publishers, leaving behind an intellectual heritage that deserves to be better known. 


\section{MARGARET HUGHES}

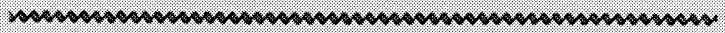 \\ LES LAURIERS SONT COUPES...

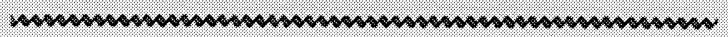

JOURNAL D'UNE VOLONTAIRE

AMERICAINE EN FRANCE-

(Avril-Septembre, 1940)

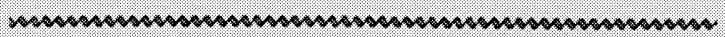

ÉDTrTONS VARTÉTÉS . MONTREAL

I. Les lauriers sont coupés. .., journal d'une volontaire américaine en France, avril-septembre I940 (Montréal: Éditions Variétés, 194I), 25I p.; (New York: Brentano's, 194I), 25 I p. 


\section{MARGARET HUGHES}

\section{LES LAURIERS SONT COUPES...}

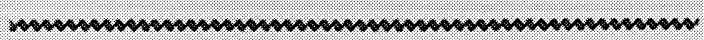

JOURNAL DUUNE VOLONTAIRE AMÉRICAINE EN FRANCE-

(Avril-Septembre, 1940 )

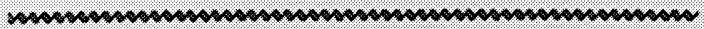
BRENTANO' : NEW YORX 


\section{GEORGES BERNANOS}
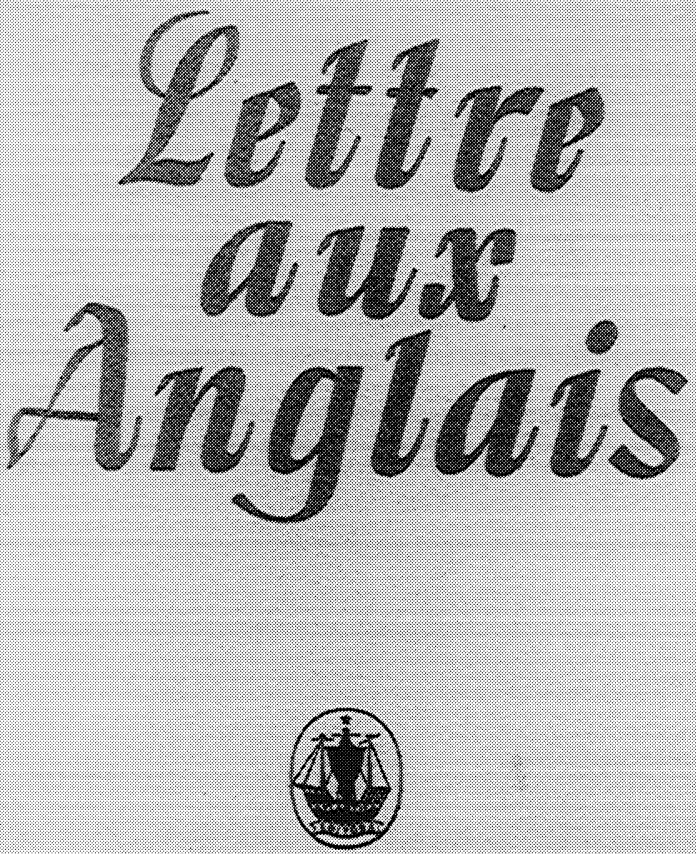

L'A RBR E

2. Georges Bernanos, Lettre aux Anglais (Rio de Janeiro: L'Arbre, Atlantica Editora, I942), 303 p. 


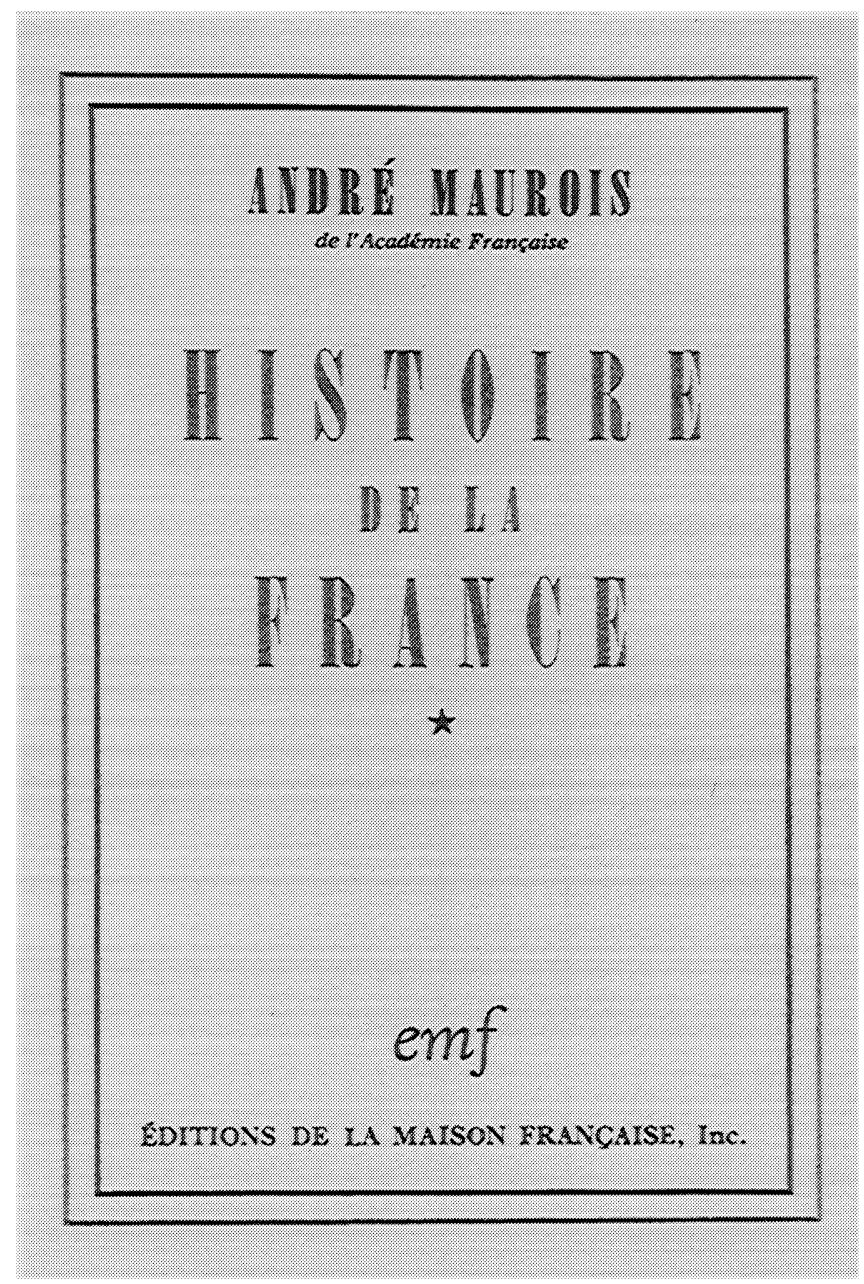

3. André Maurois, Histoire de la France (New York: Éditions de la Maison française, I947), 372 p. 
Tableau I

Production des EMF et de Brentano's classée par sujet

\begin{tabular}{|c|c|c|c|c|c|}
\hline Catégories & B'tano's & $E M F$ & Total & $\%$ & \\
\hline $\begin{array}{l}\text { GÉNÉRALITÉs } \\
\text { Dict. Encyclo. } \\
\text { Revues, annuaires }\end{array}$ & 2 & 7 & $\begin{array}{l}2 \\
7\end{array}$ & $\begin{array}{l}0.6 \% \\
2.4 \%\end{array}$ & $3.1 \%$ \\
\hline PHILOSOPHIE & 3 & 8 & II & $3.8 \%$ & $3.8 \%$ \\
\hline RELIGION, SPIRITUALITÉ & & 3 & 3 & $1.0 \%$ & $1.0 \%$ \\
\hline $\begin{array}{l}\text { SCIENCES SOCIALES } \\
\text { Sociologie, Démographie } \\
\text { Politique } \\
\text { Économie } \\
\text { Droit, Législation } \\
\text { Administration publique } \\
\text { Aide sociale, Assurances }\end{array}$ & $\begin{array}{l}\text { I } \\
5 \\
2\end{array}$ & $\begin{array}{l}3 \\
2 \\
2 \\
1\end{array}$ & $\begin{array}{l}\text { I } \\
8 \\
2 \\
2 \\
2 \\
\text { I }\end{array}$ & $\begin{array}{l}0.3 \% \\
2.8 \% \\
0.7 \% \\
0.7 \% \\
0.7 \% \\
0.3 \%\end{array}$ & $5.5 \%$ \\
\hline $\begin{array}{l}\text { SCIENCES APPLIQUÉES } \\
\text { Sciences médicales } \\
\text { Psychiatrie, Psycha. Sex }\end{array}$ & I & $\mathbf{I}$ & $\begin{array}{l}\text { I } \\
\text { I }\end{array}$ & $\begin{array}{l}0.3 \% \\
0.3 \%\end{array}$ & $0.7 \%$ \\
\hline $\begin{array}{l}\text { BEAUX-ARTS, LOISIR } \\
\text { Histoire de l'art } \\
\text { Arts décoratifs } \\
\text { Peinture }\end{array}$ & $\begin{array}{l}3 \\
\mathrm{I}\end{array}$ & 2 & $\begin{array}{l}3 \\
1 \\
2\end{array}$ & $\begin{array}{l}1.0 \% \\
0.3 \% \\
0.7 \%\end{array}$ & $2.1 \%$ \\
\hline $\begin{array}{l}\text { LITTÉRATURE } \\
\text { Histoire littéraire } \\
\text { Poésie } \\
\text { Théâtre } \\
\text { Roman } \\
\text { Essai } \\
\text { Livres pour la jeunesse }\end{array}$ & $\begin{array}{c}7 \\
4 \\
5 \\
29 \\
8 \\
13\end{array}$ & $\begin{array}{c}7 \\
5 \\
\text { I } \\
37 \\
14\end{array}$ & $\begin{array}{c}14 \\
9 \\
6 \\
66 \\
22 \\
13\end{array}$ & $\begin{array}{c}4.8 \% \\
3.1 \% \\
2.1 \% \\
22.8 \% \\
7.6 \% \\
4.5 \%\end{array}$ & $45.0 \%$ \\
\hline $\begin{array}{l}\text { GEOGRAPHIE, HISTOIRE } \\
\text { Géo., Voyages } \\
\text { Biographies, Généalogie } \\
\text { Histoire, Civilisations } \\
\text { Documents d'histoire } \\
\text { Faits Divers, Actualité } \\
\text { Armées }\end{array}$ & $\begin{array}{c}\text { I } \\
6 \\
4 \\
\\
35 \\
\text { I }\end{array}$ & $\begin{array}{c}3 \\
4 \\
8 \\
3 \\
3 \mathrm{I} \\
2\end{array}$ & $\begin{array}{c}4 \\
10 \\
12 \\
3 \\
66 \\
3\end{array}$ & $\begin{array}{c}1.4 \% \\
3.5 \% \\
4.2 \% \\
1.0 \% \\
22.8 \% \\
1.0 \%\end{array}$ & $34.0 \%$ \\
\hline NON DISPONIBLES & 5 & 9 & I4 & $4.8 \%$ & $4.8 \%$ \\
\hline TOTAL & 136 & 153 & 289 & $100.0 \%$ & $100.0 \%$ \\
\hline
\end{tabular}


I8 I Michon : Les éditeurs de littérature française aux États-Unis

Tableau II

Éditeurs français de New York, production annuelle

\begin{tabular}{c|c|c|c} 
& Brentano's & $E M F$ & Total \\
\hline I940 & - & 7 & 7 \\
\hline I94I & 9 & 23 & 32 \\
\hline I942 & I I & I7 & 28 \\
\hline I943 & 8 & II & I9 \\
\hline I944 & I0 & I I & 2I \\
\hline I945 & I6 & I3 & 29 \\
\hline I946 & 3 & 3 & 6 \\
\hline I947 & 4 & 2 & 6 \\
\hline Datés & 6 I & 87 & I48 \\
Non datés & 75 & 66 & I4I \\
\hline TOTAL & I36 & I53 & 289
\end{tabular}

Tableau III

Production en français des éditeurs latino-américains

\begin{tabular}{l|c} 
MEXICO & \\
Quetzal & I5 \\
\hline RIO DE JANEIRO & \\
Americ-Edit & 95 \\
Atlantica Editora $\mid 26$ & 26 \\
\hline BUENOS AIRES & \\
Sur & IO \\
Hachette & I8 \\
Viau & 5 \\
\hline TOTAL & I69
\end{tabular}




\section{Graphique I}

\section{EMF et Brentano's}

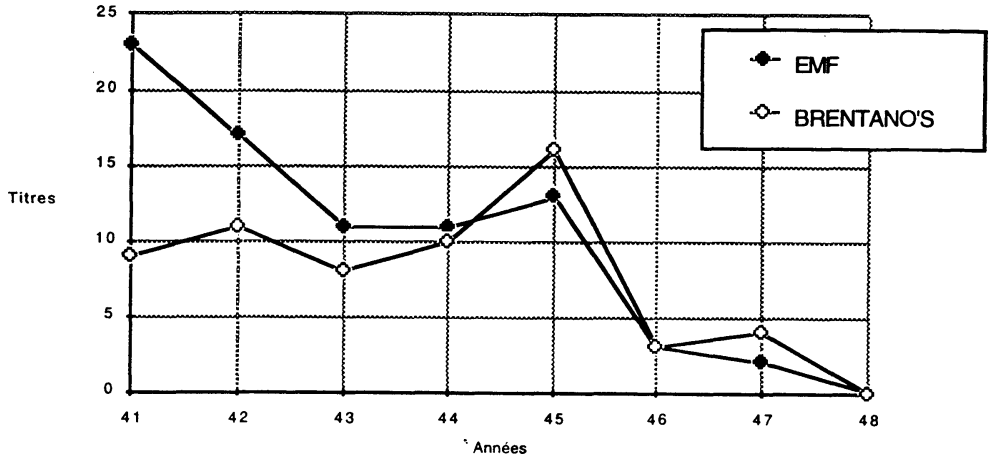

NOTES

I D'après le témoignage de Claude Hurtubise, directeur des Éditions de l'Arbre (194 I-I948), recueilli par Jacques Michon en 1987, et les procès-verbaux de la Société des éditeurs canadiens de langue française pour l'année 1945, Fonds Luc Lacroix, GRELQ, Université de Sherbrooke.

2 Concernant ce projet voir l'article que nous avons publié avec notre collègue Richard Giguère, "An Approach to the History of Publishing: TwentiethCentury Québec," Book Research Quarterly 6, no I (Spring I990): 55-64.

3 D'après une compilation effectuée par nous à partir des "Notices en langue française" du Canadian Catalog of Books, 1940-49, rééditées dans Notices en langue française du Canadian Catalog of Books, I92I-1949, avec index établi par Henri Bernard Boivin (Montréal: Bibliothèque nationale du Québec, 1975), XII-263-I99 p.

4 Pour plus de détails sur cette question, voir, entre autres, Éditeurs transatlantiques, études publiées sous la direction de Jacques Michon (Sherbrooke, Québec: Ex Libris, 1991), 245 p.

5 D'après les “Notices en langue française" du Canadian Catalog of Books, 1940-49.

6 “L'industrie du livre français au Canada», Notre temps (Montréal), I9 janvier 1946, p. 3 .

7 Voir à ce sujet les travaux de Pascal Fouché et en particulier L'édition française sous l'occupation, 1940-1944 (Paris: Bibliothèque de Littérature françaises 
contemporaine de l'Université de Paris 7, 2 vol.) 453, 457 p., et de Jean-Michel Guiraud, La vie intellectuelle et artistique à l'époque de Vichy et sous l'occupation, 1940-1944 (Marseille: CRDP, 1987), $356 \mathrm{p}$.

8 Nous excluons ici une entreprise comme F.S. Croft \& Co. qui réimprimait les ouvrages de la maison Larousse dont elle était le distributeur aux États-Unis.

9 Le Canada, en guerre depuis 1939, avait adopté un Arrêté exceptionnel qui permettait de rééditer sur son territoire tous les ouvrages des pays ennenis, ce qui était le cas de la France occupée, dès juin 1940, alors que les États-Unis n'avaient pas adopté une pareille mesure. On sait que les États-Unis n'entreront en guerre qu'en décembre $194 \mathrm{I}$.

Io Quelques nouveautés réussissaient à traverser l'Atlantique. Voir, entre autres, Le Crève-coeur d'Aragon (Paris: coll. Métamorphoses, XI, Gallimard, I94I), [Montréal: Éditions Variétés, 1943], 73 p.; Vercors, Les Silences de la mer [sic] (New York: Pantheon Books, Éditions Jacques Schiffrin, I943), 68 p.; et les deux derniers volumes de la "Chronique des Pasquier" publiés en feuilleton dans $L a$ Revue des Deux Mondes en I 940 et I94 I et réédités en deux volumes à Montréal en 1943: Suzanne et les jeunes hommes, [Montréal: Éditions de l'Arbre, 1943], 283 p. et La passion de Joseph Pasquier (Montréal: Éditions de l'Arbre, I943).

I I Adresse: Éditions de la Maison française, Inc., 6ro Fifth Avenue, New York.

I2 Maurois, Tragédie en France, coll. "Voix de France» (New York: Éditions de la Maison française, r940), 23 I p.

I3 Mémoires II, les années de travail (New York: EMF, I942), p. 25 I.

I4 L'éditeur publiait aussi annuellement un recueil de mélanges, intitulé Les Oeuvres nouvelles, qui rassemblaient des nouvelles (short stories), des récits vécus, des souvenirs et des essais d'intérêt général.

I5 Voir Catalogue général, 1940-1944 (New York: Éditions de la Maison française, s.d.), I 8-b.

I6 Pour une présentation de cette série, voir Paul Bleton, “Insolite: l'espionnage d'un poète," Les Cahiers de l'imaginaire (Laillé, France) no 27-28 (I989): 7 I-7.

17 Concernant les éditions américaines de Saint-Exupéry, voir Écrits de guerre, 1939-1944, avec la Lettre à un otage, et des témoignages et documents, préface de Raymond Aron (Paris: Gallimard, 1982), 652 p.

I8 Adresse: Brentano's, Inc., 586 Fifth Avenue, New York, N.Y.

I9 “Les Éditions françaises Brentano's," Renaissance, New York, Brentano's, vol. II-III, I 944-45, p. 55 I.

20 Voir Nicolas Politis, La morale internationale, préface de Robert Tenger, "Bibliothèque Brentano's, études historiques, économiques et sociales," (New York: Brentano's, I 944/ couverture p. 4. Il y aurait une étude à faire sur le rôle de Robert Tenger, vraisemblablement le directeur littéraire de la maison, qui signe une grande partie des préfaces des ouvrages figurant au catalogue de la maison d'édition en particulier celles de cette collection.

2I Breton, Arcane 17, note de l'éditeur Robert Tenger (New York: Brentano's, 1945), I 77 p. Au sujet de la composition de ce livre, voir Suzanne Lamy, André Breton, hermétisme et poésie dans Arcane 17 (Montréal: Presses de l'Université de Montréal, 1977), 268 p. 
22 Denis de Rougemont, La part du diable (New York: Brentano's; Montréal: Éditions Bernard Valiquette, I942), I95 p. Au sujet de cette édition, voir Denis de Rougemont, Journal d'une époque, 1926-1946 (Paris: Gallimard, 1968): 5267.

23 Parmi les ouvrages publiés au Canada et distribués par Brentano's, signalons le Moyen âge, survivances et réveil d'Henri Focillon (Valiquette, 1943) et plusieurs romans canadiens publiés ou réédités par les Éditions Variétés et Bernard Valiquette (Trente arpents de Ringuet, Les Velder de Robert Choquette), et sur les presses du Devoir (Léo-Paul Desrosiers, Sources, Les Opiniâtres; Michelle Le Normand, La maison aux phlox, ze édition). Alors que la plupart des livres édités aux EMF sont imprimés à New York, chez Albert Martin Inc., plusieurs livres de Brentano's sont imprimés à Montréal.

24 Les lauriers sont coupés. . ., journal d'une volontaire américaine en France, avril-septembre I940 (Montréal: Éditions Variétés, I94I), 25 I p.; (New York: Brentano's, I94I), 25 I p.

25 Catalogue général, 1940-1944 (New York: ÉMF, s.d.), I-2.

26 Ce sont, d'après nos relevés, Raoul Aglion, Fernand Corcos, Michel GeorgesMichel, Robert Goffin, Pierre de Lanux, André Maurois, B. Mirkine-Guetzevitch, Antoine de Saint-Exupéry et Louis Verneuil.

27 On sait que les EMF signent des contrats d'exclusivité avec ses auteurs. Voir la lettre de Raissa Maritain à Guy Sylvestre, le I7 décembre I94 I, Écrits du Canada français no 49 (1983): 99 .

28 Certains de ces auteurs publièrent aussi des livres à Montréal et chez d'autres éditeurs new yorkais, comme Pantheon Books et Didier, qui étaient aussi des entreprises créées par des réfugiés européens et que nous n'avons pas le temps d'aborder ici. Contrairement aux EMF, à Brentano's et aux éditeurs canadiens, Pantheon et Didier publient aussi des livres en anglais, nouveautés et traductions de classiques européens, qui constituent une part importante de leur production.

29 Tebbel, A History of Book Publishing in the United States, vol. 4, The Great Change, 1940-1980 (New York: Bowker, I98 r), 45. Didier s'installera à Montréal dans les années 50, de même que le Cercle du livre de France, un club du livre francophone créé à New York en 1947 qui deviendra un des principaux éditeurs de littérature canadienne-française au Québec dans les années 50 et 60 .

30 "The export markets were far-flung indeed, especially as one area after another was liberated: French North Africa and the Congo; Egypt, Syria, Lebanon; Mozambique, Madagascar; Latin America; French communities all over America; Indo-China and the Far-East," "French Canadian Publishing: A Rapid Development," Publishers Weekly, I4 September 1946, p. I346.

3 I Voir les procès-verbaux de l'année I 945 (en particulier celui du 30 mai 1945) de la Société des éditeurs canadiens du livre français, où l'on voit que la Société sert $\mathrm{d}$ 'intermédiaire entre les éditeurs et le gouvernement pour l'expédition de livres en Algérie pour une valeur de 25 848.74 \$ dollars canadiens. Fonds Luc Lacroix, Archives du GRELQ, Université de Sherbrooke.

32 Renseignements fournis par Claude Hurtubise lors d'une entrevue avec Jacques 
Michon et Richard Giguère le Ier septembre I986, qui confirment les propos recueillis par Silvie Bernier auprès de Paul Péladeau (Éditions Variétés, Montréal) en décembre 1984 .

33 Adresse: Ediciones Quetzal, Pasaje Iturbide I8, Mexico, D.F.

34 René Etiemble était à l'Université de Chicago depuis I934. Berveiller, gaulliste réfugié au Mexique, était un ancien condisciple $\mathrm{d}^{\prime}$ Etiemble à $\mathrm{l}^{\prime}$ Ecole normale supérieure (Paris).

35 Caillois, La communion des forts, études de sociologie contemporaine, coll. “Renaissance» (Mexico: Ediciones Quetzal, S.A.; Montréal: Éditions Bernard Valiquette, 1943l, i54 p.

36 A ce sujet, voir Etiemble et Jules Supervielle, Correspondance, 1936-1959, édition critique, texte établi, annoté, préfacé par Jeannine Etiemble (Paris: Société d'enseignement supérieur, 1969|, 200 p. Voyant ses projets compromis chez Quetzal, après le départ de son ami Berveiller, Etiemble songe un moment à présenter ses projets de publications aux Éditions de l'Arbre de Montréal (p. ro9-ro). L'Arbre publiera de lui une traduction de Goliath or the March of Fascism (1937) de G.A. Borgese sous le titre de La Marche du fascisme (Montréal: L'Arbre, 1945, 265 p.). Victor Serge, exilé au Mexique, également édité chez Quetzal (Hitler contra Stalin, I94 I) sera lui aussi publié aux Éditions de l'Arbre; il y fera paraître son roman intitulé Les Derniers temps (Montréal: L'Arbre, 1946, 2 vol., $26 \mathrm{I}-258 \mathrm{p}$ ).

37 Comme on peut le constater sur la page de couverture de l'édition canadienne de La communion des forts, études de sociologie contemporaine.

38 Adresse: Americ-Edit, Caixa Postal 429, Rio de Janeiro, Brésil.

39 L'Almanach des lettres 1947 (Paris: Ed. de Flore et la Gazette des lettres, I946), résume la situation ainsi: “Au Canada, les Éditions Variétés, les Éditions de l'Arbre, les Éditions Bernard Valiquette ont publié de nombreux volumes de Duhamel et Mauriac notamment, dans la présentation même et sous la firme des éditeurs français, avec la mention Imprimé au Canada. Une loi a permis cette opération en excluant notamment le Canada des accords de la Convention de Berne. Les droits d'auteur et d'éditeur, ramenés uniformément à ı०\%, devaient subir la déduction de l'impôt sur le revenu. [ . . . E En Amérique du Sud, un problème analogue à celui du Canada s'est posé. Il a été résolu par une loi du 23 octobre 1943, votée à Alger et semblable à la loi canadienne: autorisation de réimpression moyennant réserve d'un pourcentage pour l'éditeur français propriétaire. De I $6 \%$, ce pourcentage a été par la suite ramené à I0\% $16 \%$ pour l'auteur, $4 \%$ pour l'éditeur). C'est principalement la Société Americédit, dont le directeur littéraire était l'écrivain français Max Fischer, qui, à Rio de Janeiro, a publié des oeuvres de Mauriac, Colette, Anatole France, Loti, Charles Seignobos, etco' (p. 153).

40 Adresse: Atlantica Editora, Praça Getulo Vargas 2, Rio de Janeiro, Brésil.

4I Bernanos, Lettre aux Anglais (Rio de Janeiro: L'Arbre, Atlantica Editora, 1942), $303 \mathrm{p}$. (voir illustration).

42 Ils seront réédités en un volume en 1987: Le chemin de la Croix-des-âmes, nouvelle édition, complétée, corrigée et annotée par Brigitte et Jean-Loup Berna- 
nos, d'après les manuscrits. Augmentée de tous les articles et messages écrits au Brésil, coll. «NRP, Littérature» (Monaco: Éditions Le Rocher, 1987) 904 p.

43 Le Comte de Lautréamont, Les chants de Maldoror (Buenos Aires: Viau, 1944), 252 p.; Stéphane Mallarmé, Les poèmes d'Egar Poe, ill. de Raquel Forner (Buenos Aires: Viau, I944), I 22 p. Nous avons aussi retrouvé un récit de Joseph Kessel publié sous la raison sociale de Viau-Feugère: Les Maudru (Buenos Aires: ViauFeugere, I 945 l, I 39 p. qui annonce, sur la quatrième de couverture, la publication de cinq autres titres, dont deux vraisemblablement parus, Le champ de la mort lente de Jean Jacques Bernard, Péguy ( 2 vols) de Romain Rolland, et trois autres à paraître: Les poètes de la Résistance (anthologie), Vercors intime et Le droit romain n'existe plus d'Aragon.

44 Bory, Mon village à l'heure allemande (New York: Éditions de la Maison française, 1945l, $309 \mathrm{p}$.

45 Sur cette question voir Yvan Cloutier, "Sartre en quête d'un éditeur francophone en Amérique," The French Review 66, no 5 (April 1993): 752-9. La fin de la guerre donne aussi lieu à un autre type de publications, celles des écrivains interdits en France pour cause de collaboration (ex. Pierre Benoit, Jamrose, Albin Michel, I945, imprimé au Canada par les Éditions J.-A. Pony). D'autres, comme Michel Mohrt, soupçonnés de complaisance avec l'ennemi, traverseront l'Atlantique en 1945, le temps de se faire oublier. Voir à ce sujet le roman de Michel Mohrt, Mon royaume pour un cheval (Paris: Albin Michel, Le Livre de poche, 1968), 445 p., où l'auteur évoque ses années d'exil au Canada et son travail dans une maison d'édition montréalaise.

46 Cette situation crée un certain optimiste chez les éditeurs du Canada et d'Amérique latine. Dans un dialogue radiophonique diffusé à Montréal en 1946, Viau de Buenos Aires et Lucien Parizeau, l'éditeur de Montréal, tiennent les propos suivants: “- Viau: Je suis d'ailleurs venu précisément au Canada pour prendre contact avec les Sociétés, ou les Chambres coopératives, des éditeurs. Je crois qu'en unissant les efforts des deux hémisphères nous pouvons agrandir le domaine de la culture française ... - Parizeau: Sans compter, et ce n'est pas un point de vue secondaire, que cela permet à l'écrivain, au romancier, au poète, au philosophe, de connaître des tirages auxquels il n'eût jamais rêvé . . . Les éditeurs français lui assurent les marchés européens; nous lui assurons ici les marchés américains, grâce au réseau de distribution déjà établi." Transcription manuscrite (inédite), Fonds Lucien Parizeau, Archives du GRelQ, Université de Sherbrooke. La Libreria Hachette à Buenos Aires, dont nous n'avons pas le temps de parler ici, qui publie 18 titres entre 1944 et 1946, semble avoir été créée justement pour répondre à ce besoin de diffusion en Amérique latine.

47 Les grandes amitiés (New York: EMF, I94I-44) de Raissa Maritain sont distribuées à Paris par Desclée de Brouwer et Le nouveau Sphinx de Robert Goffin (New York: Brentano's, I94I) est distribué par Flammarion. Attestés par les marques de distributeurs imprimées sur les exemplaires que nous avons consultés.

48 Les statistiques de la production littéraire de la même époque (voir J. Michon dans Littérature, no 66, mai 1987, p. I I8) et dans une entreprise comme les 


\section{I87 Michon : Les éditeurs de littérature française aux États-Unis}

éditions de l'Arbre (voir Éditeurs transatlantiques, cité plus haut, p. I 7) viennent confirmer cette tendance.

49 On pourrait appliquer au Québec, cette remarque de Victoria Ocampo: «Notre Amérique est un pays à découvrir et rien ne nous incite plus à cette découverte, rien ne nous met mieux sur la piste de notre vérité, que la présence, l'intérêt, la curiosité, les réactions de nos amis d'Europe", cité dans La revue des revues, no 5 (printemps 1988): 26. Sur les relations franco-américaines dans le domaine de l'édition, voir Paris-New York, échanges littéraires au vingtième siècle, exposition 9 juin au I2 septembre I977, Paris, B.P.I., Centre National d'Art et de Culture Georges Pompidou, 1977, I 33 p. 
